\title{
CORRECTION
}

\section{Correction to: The accuracy and accessibility of cited evidence: a study examining mental health policy documents}

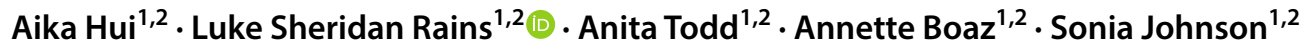

Published online: 11 November 2019

(c) The Author(s) 2019

\section{Correction to: Social Psychiatry and Psychiatric Epidemiology https://doi.org/10.1007/s00127-019-01786-8}

In the original publication of the article there was an error in the results section of the abstract. The first sentence should have read:

"Of the 236 statements, 141 (59.7\%) accurately represented the referenced source, 45 (19.1\%) contained major errors and $50(21.2 \%)$ contained minor errors in accuracy."

The original article was corrected.

The original article can be found online at https://doi.org/10.1007/ s00127-019-01786-8.

Luke Sheridan Rains

1.sheridanrains@ucl.ac.uk

Aika Hui

uctvahu@ucl.ac.uk

Anita Todd

anita.todd.15@ucl.ac.uk

Annette Boaz

a.boaz@sgul.kingston.ac.uk

Sonia Johnson

s.johnson@ucl.ac.uk

1 Division of Psychiatry, University College London,

London W1T 7NF, UK

2 Centre for Health and Social Care Research, Kingston

University and St George's University of London,

London KT1 2EE, UK 